\begin{tabular}{|c|l|}
\hline Title & Estimation of Predictability with a Newly Derived Index to Quantify Similarity among Ensemble Members \\
\hline Author(s) & Y amada, Tomohito J.; Koster, Randal D.; Kanæe, Shinjiro; Oki, Taikan \\
\hline Citation & $\begin{array}{l}\text { Monthly Weather Review, 135(7), 2674.2687 } \\
\text { https://doi.org/40.1175MWR3418.1 }\end{array}$ \\
\hline Issue Date & 2007-07 \\
\hline Doc URL & http://hdl.handle.net/2115/52269 \\
\hline Type & article \\
\hline File Information & MWR135_2674_2687.pdf \\
\hline
\end{tabular}

Instructions for use 


\title{
Estimation of Predictability with a Newly Derived Index to Quantify Similarity among Ensemble Members
}

\author{
TOMOHITO J. YAMADA \\ Institute of Industrial Science, University of Tokyo, Tokyo, Japan \\ RANDAL D. KOSTER \\ NASA Goddard Space Flight Center, Greenbelt, Maryland \\ SHINJIRO KANAE \\ Research Institute for Humanity and Nature, Kyoto, and Institute of Industrial Science, University of Tokyo, Tokyo, Japan \\ TAIKAN OKI \\ Institute of Industrial Science, University of Tokyo, Tokyo, Japan
}

(Manuscript received 22 December 2005, in final form 11 October 2006)

ABSTRACT

\begin{abstract}
This study reveals the mathematical structure of a statistical index, $\Omega$, that quantifies similarity among ensemble members in a weather forecast. Previous approaches for quantifying predictability estimate separately the phase and shape characteristics of a forecast ensemble. The diagnostic $\Omega$, on the other hand, characterizes the similarity (across ensemble members) of both aspects together with a simple expression. The diagnostic $\Omega$ is thus more mathematically versatile than previous indices.
\end{abstract}

\section{Introduction}

Correlation functions and coefficients have been used extensively to quantify the phase (correlation) similarity between time series of ensemble members (hereafter, EMs). Taylor (1920) first described the physical concepts, and Wiener (1930) completed the theory foundations using general harmonic analysis. In addition, the autocorrelation function is transformed into the power spectrum through Fourier transform analysis (Wiener-Khintchine's formula; Taylor 1938). Various studies have established and described mathematical and physical correlations between two EMs. These studies have advanced our understanding of such meteorological phenomena as turbulence and chaos.

Thompson (1957) originally noted the sensitivity of large-scale atmospheric patterns to initial conditions.

Corresponding author address: Tomohito J. Yamada, Be 607, Institute of Industrial Science, University of Tokyo, 4-6-1 Komaba, Meguro-ku, Tokyo 153-8505, Japan.

E-mail: tomohito@iis.u-tokyo.ac.jp
Lorenz (1963) suggested that a nonperiodic evolution occurs in three types of simultaneous, ordinary, differential equations, even if initial conditions have subtle differences. Because of the atmosphere's chaotic behavior, a deterministic numerical forecast with a single atmospheric initial condition may have limited value for prediction. An ensemble forecast consisting of a number of individual forecast simulations, each simulation using slightly different initial conditions, can gauge and reduce the prediction errors that arise from chaotic behavior. Miyakoda et al. (1986), for example, used a global, nine-level general circulation model (GCM) and found that ensemble mean forecasts, when validated against observations, had smaller root-meansquare (RMS) errors and larger anomaly correlation scores than did individual forecasts. Ensemble forecasts are a practical approximation of the general stochastic dynamic prediction method (Epstein 1969); see Lewis (2005) for further details. The correlations among the members, the RMS differences between the members, and the RMS departure from the ensemble mean are all used to characterize ensemble spread and to estimate

DOI: $10.1175 / M W R 3418.1$

(C) 2007 American Meteorological Society 
forecast skill or probability distributions in mediumrange weather forecasts. [Such ensemble forecasts are provided routinely by meteorological centers such as the National Centers for Environmental Prediction (NCEP), the European Centre for Medium-Range Weather Forecasts (ECMWF), and the Japan Meteorological Agency (JMA; Murphy 1988; Kimoto et al. 1992).] Statistical diagnostics derived from forecast ensembles have also been used to estimate potential predictability at seasonal time scales (Rowell et al. 1995; Stern and Miyakoda 1995; Sugi et al. 1997; Rowell 1998; Phelps et al. 2004). For example, Rowell et al. (1995) examined the impact of internal atmospheric variability and sea surface temperature (SST) forcing on predictability over tropical North Africa using a signal-tonoise ratio from analysis of variance (Scheffe 1959). Shukla et al. (2000) used the signal-to-noise ratio along with anomaly correlation coefficients to suggest that winter mean circulation anomalies over the PacificNorth American region were highly predictable during years of large tropical SST anomalies.

The individual members of an ensemble of mediumrange or seasonal forecasts can differ in two key ways: 1) in their "shape" by which we mean their mean values and the amplitudes of their temporal variations, and 2) in their "phase," as characterized by their temporal correlation. If all EMs are completely correlated, phase predictability is by definition perfect, even if mean values and amplitudes vary between the EMs. In contrast to the correlation diagnostic, RMS differences can represent the difference of mean values and amplitudes among EMs for each time period including the difference of period characteristics. Therefore the RMS differences estimate the "shape predictability" among EMs over a given period-conceivably, the RMS difference can be low even if the EMs are completely uncorrelated. A unified diagnostic for the statistical evaluation of predictability-one that examines both the phase and shape elements - is not in standard use. (Note that throughout this text, the term "predictability" is used to describe the degree to which initial conditions affect some aspect of a forecast ensemble, no matter if the forecast agrees with observations or not. This is in contrast to "predictive skill," which implies a comparison to observations.)

Koster et al. (2000, 2002, hereafter K02) use a statistical index, $\Omega$, that does measure the degree of similarity between EMs in both phase and shape. According to $\mathrm{K} 02, \Omega$ measures the ratio of signal variance to total variance, much like the aforementioned diagnostic used by Rowell et al. (1995). Many recent studies (Koster et al. 2004, 2006; Dirmeyer et al. 2006; Guo et al. 2006) have used the $\Omega$ diagnostic to estimate the coupling strength between soil moisture and precipitation variability. These particular studies were performed as part of the Global Land-Atmosphere Coupling Experiment (GLACE), a project sponsored by the Global Energy and Water Cycle Experiment (GEWEX) and the Climate Variability program (CLIVAR). Through joint analysis of the results of a dozen atmospheric general circulation models (AGCMs), GLACE found large degrees of coupling strength (for boreal summer) over the Great Plains of North America, India, and the Sahel (Koster et al. 2004).

The present paper provides a description of the mathematical structure of $\Omega$ to illustrate all elements of its usefulness for ensemble forecast analysis. Section 2 shows how $\Omega$ is calculated, and section 3 provides two separate mathematical interpretations of $\Omega$. The behavior of $\Omega$ is explored in section 4 for some idealized, hypothetical situations. Section 5 then introduces a new application of $\Omega$-its use in evaluating the predictability among ensemble members in medium-range forecasts.

\section{Definition of $\Omega$}

The output (e.g., temperature, precipitation) from an ensemble forecast can be expressed as a matrix $\mathbf{A}$ :

$$
\mathbf{A}=\left(\begin{array}{cccc}
x_{11} & x_{12} & \cdots & x_{1 n} \\
x_{21} & x_{22} & \cdots & x_{2 n} \\
\vdots & \vdots & \ddots & \vdots \\
x_{m 1} & x_{m 2} & \cdots & x_{m n}
\end{array}\right),
$$

where $x_{i j}$ is a variable averaged over $n$ time periods $(j=$ $1,2, \ldots, n)$ for each of $m$ EMs $(i=1,2, \ldots, m)$. Two variances are calculated. The ensemble mean is computed with (1), and the temporal variance of the ensemble mean $\left(\sigma_{b}^{2}\right)$ is computed with (2) (see Fig. 1a):

$$
\begin{aligned}
b_{j} & =\frac{1}{m} \sum_{i=1}^{m} x_{i j}, \\
\sigma_{b}^{2} & =\frac{1}{n} \sum_{j=1}^{n}\left(b_{j}-\bar{x}\right)^{2} .
\end{aligned}
$$

Here, $\bar{x}$ is the temporal mean of $b_{j}$, calculated with

$$
\bar{x}=\frac{1}{n} \sum_{j=1}^{n} b_{j} .
$$

Next, the full-sample variance $\left(\sigma^{2}\right)$ is calculated across all time periods and all EMs (Fig. 1b):

$$
\sigma^{2}=\frac{1}{m n} \sum_{i=1}^{m} \sum_{j=1}^{n}\left(x_{i j}-\bar{x}\right)^{2} .
$$



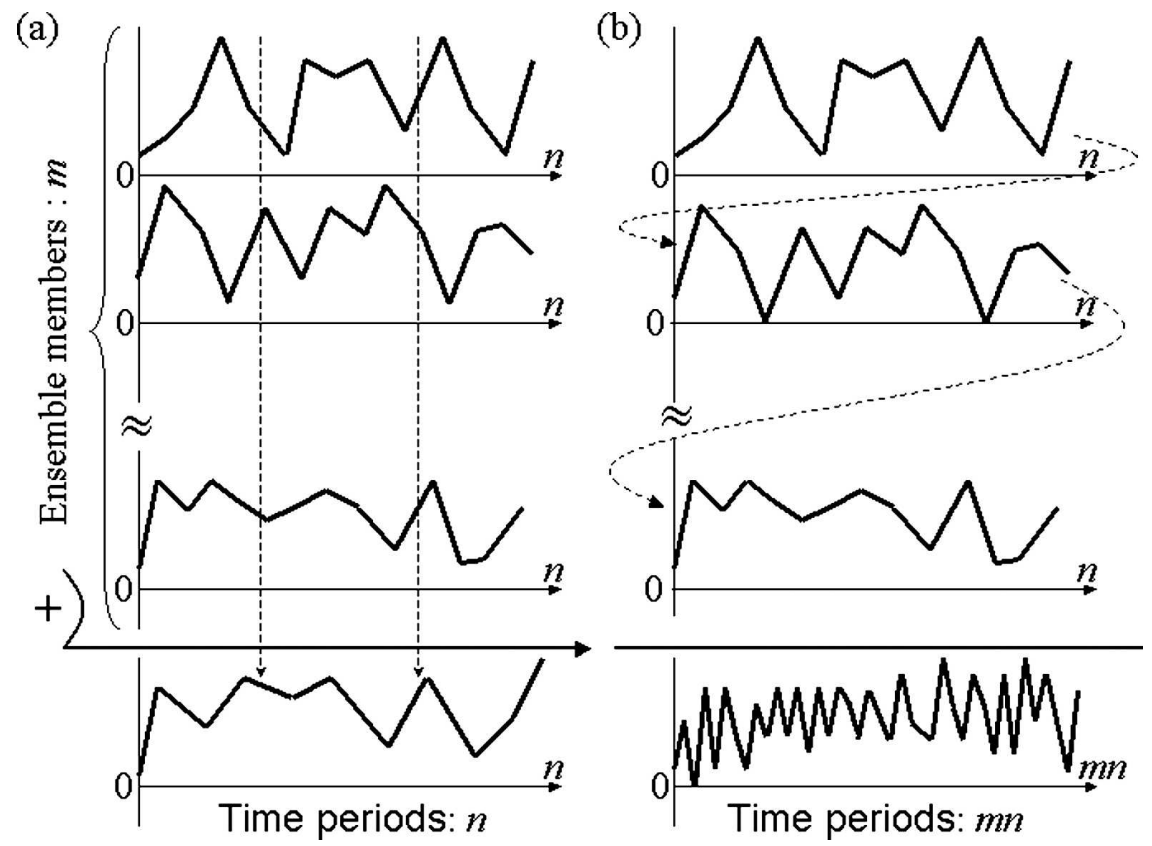

FIG. 1. Two types of variances for calculating statistical index $\Omega$. (a) The temporal variance of the ensemble mean $\left(\sigma_{b}^{2}\right)$ is calculated by time the ensemble mean $\left(b_{j}\right)$. (b) The full-sample variance $\left(\sigma^{2}\right)$ is calculated across all time periods and EMs.

The statistical index $\Omega$ is then defined as

$$
\Omega=\frac{m \sigma_{b}^{2}-\sigma^{2}}{(m-1) \sigma^{2}} .
$$

K02 noted that if each EM produces exactly the same time series, then $\sigma_{b}^{2}$ equals $\sigma^{2}$, and $\Omega$ will be 1 . In contrast, if all EMs are completely uncorrelated, then $\sigma_{b}^{2}$ approaches $\left(\sigma^{2} / m\right)$, and $\Omega$ will be approximately 0 . Thus, outside of sampling error, $\Omega$ varies from 0 to 1 . Values closer to 1 indicate a greater degree of similarity amongst the EMs.

\section{Mathematical analyses of $\Omega$}

\section{a. Mathematical analysis based on idealized normalizations}

This section describes our first interpretation of the mathematical structure of $\Omega$. Consider a generalized case of $m$ EMs, each with $n$ time periods; $X_{i j}$ is a simulated variable (e.g., temperature or precipitation amount), and $F_{j}$ is a forcing (e.g., SST or soil moisture) that varies with time. The $F_{j}$ time series is the same for each ensemble member.

Variables $X_{i j}$ and $F_{j}$ can be normalized as follows:

$$
x_{i j}=\frac{X_{i j}-\bar{X}}{\sigma_{X}},
$$

$$
f_{j}=\frac{F_{j}-\bar{F}}{\sigma_{F}},
$$

where $\bar{X}$ is the temporal ensemble mean of $X_{i j}$, and $\bar{F}$ is the temporal mean of $F_{j}$. Also, $\sigma_{X}$ and $\sigma_{F}$ represent the standard deviations of $X_{i j}$ and $\bar{F}$ across all time periods and all EMs.

Consider that the value of any simulated variable $x_{i j}$ is controlled in part by the forcing term $f_{j}$ and by chaotic variability, represented by the random normal deviate $\xi_{i j}$. The variable $x_{i j}$ can be rewritten as

$$
x_{i j}=\rho f_{j}+\sqrt{1-\rho^{2}} \xi_{i j} .
$$

The term $\rho$ is effectively the correlation coefficient between the variable and the underlying boundary forcing that controls it. By definition,

$$
\bar{x}_{i}=\bar{f}=\bar{\xi}_{i}=0
$$

and

$$
\sigma_{x_{i}}^{2}=\sigma_{f}^{2}=\sigma_{\xi_{i}}^{2}=1,
$$

where (9) gives mean values of $x_{i}, f$, and $\xi_{i}$, and (10) gives their variances.

The variance $\sigma_{b}^{2}$ of the ensemble mean can be estimated using (1) and (8): 


$$
\begin{aligned}
\sigma_{b}^{2}= & \frac{1}{n} \sum_{j=1}^{n} b_{j}^{2} \\
= & \rho^{2}\left(\frac{1}{n} \sum_{j=1}^{n} f_{j}^{2}\right)+\frac{2 \rho \sqrt{1-\rho^{2}}}{m n} \sum_{i=1}^{m} \sum_{j=1}^{n} f_{j} \xi_{i j} \\
& +\frac{\left(1-\rho^{2}\right)}{m^{2} n} \sum_{j=1}^{n}\left(\sum_{i=1}^{m} \xi_{i j}^{2}+2 \sum_{k=1}^{m-1} \sum_{l=k+1}^{m} \xi_{k j} \xi_{l j}\right) .
\end{aligned}
$$

Because $\xi_{i j}$ is a random variable,

$$
\begin{gathered}
\sum_{i=1}^{m} \sum_{j=1}^{n} f_{j} \xi_{i j}=\sum_{j=1}^{n} f_{j} \sum_{i=1}^{m} \xi_{i j}=0, \\
\sum_{j=1}^{n} \xi_{k j} \xi_{l j}=0 \quad \text { for } \quad k \neq l .
\end{gathered}
$$

Substituting (12) and (13) into (11) and applying (10) yields

$$
\sigma_{b}^{2}=\frac{\rho^{2}(m-1)+1}{m}
$$

Substitution of $\sigma_{b}^{2}$ from (14) into (5) yields

$$
\Omega=\rho^{2} .
$$

Thus, if a simulated variable can be divided into the forcing (e.g., SST or soil moisture) and random terms (perturbation of atmospheric behavior) and if the most simplified normalization is applied, then $\Omega$ is equivalent to $\rho^{2}$. In other words, $\Omega$ represents the square of the coefficient between the variable of interest and the boundary forcing. Key to the usefulness of $\Omega$ is that it can be computed without knowing the particular character of the boundary forcing that controls the predictability. In other words, $f$, which might represent a subtle spatial pattern of the forcing, never needs to be explicitly computed.

\section{b. Mathematical analysis with strict normalizations}

A second approach can be used to examine $\Omega$. The temporal mean $\left(a_{i}\right)$ and the temporal variance $\left(\sigma_{\mathrm{amp}_{i}}^{2}\right)$ of each ensemble member are calculated as follows:

$$
\begin{aligned}
a_{i} & =\frac{1}{n} \sum_{j=1}^{n} x_{i j}, \\
\sigma_{\mathrm{amp}_{i}}^{2} & =\frac{1}{n} \sum_{j=1}^{n}\left(x_{i j}-a_{i}\right)^{2} .
\end{aligned}
$$

Also, note that (2) can be rewritten as

$$
\sigma_{b}^{2}=\frac{1}{m^{2}} \sum_{k=1}^{m} \sum_{l=1}^{m} R_{k l}-\bar{x}^{2},
$$

where

$$
R_{k l}=\frac{1}{n} \sum_{j=1}^{n} x_{k j} x_{l j} .
$$

The mathematical structure of $\Omega$ can be derived for the strictest mathematical normalization. Consider the normalization of $x_{i j}$ by $a_{i}$ and $\sigma_{\mathrm{amp}_{i}}$, as shown in (20) and (21):

$$
\begin{gathered}
x_{k j}^{\prime}=\frac{x_{k j}-a_{k}}{\sigma_{\mathrm{amp}_{k}}}, \\
x_{l j}^{\prime}=\frac{x_{l j}-a_{l}}{\sigma_{\mathrm{amp}_{l}}} .
\end{gathered}
$$

Substitution of (20) and (21) into (19) yields

$$
\begin{aligned}
R_{k l}= & \frac{1}{n} \sum_{j=1}^{n}\left(a_{k} a_{l}+\sigma_{\mathrm{amp}_{k}} a_{l} x_{k j}^{\prime}+\sigma_{\mathrm{amp}_{l}} a_{k} x_{l j}^{\prime}\right. \\
& \left.+\sigma_{\mathrm{amp}_{k}} \sigma_{\mathrm{amp}_{l}} x_{k j}^{\prime} x_{l j}^{\prime}\right),
\end{aligned}
$$

where the sum of $x_{k j}^{\prime}$ or $x_{l j}^{\prime}$ across all time periods in each EM is 0 , that is,

$$
\begin{gathered}
\sum_{j=1}^{n} x_{k j}^{\prime}=0, \\
\sum_{j=1}^{n} x_{l j}^{\prime}=0 .
\end{gathered}
$$

Thus, (22) can be written as

$$
R_{k l}=\frac{1}{n} \sum_{j=1}^{n}\left(a_{k} a_{l}+\sigma_{\mathrm{amp}_{k}} \sigma_{\mathrm{amp}_{l}} x_{k j}^{\prime} x_{l j}^{\prime}\right) .
$$

Substitution of (25) into (18) yields

$\sigma_{b}^{2}=\frac{1}{m^{2}} \sum_{k=1}^{m} \sum_{l=1}^{m} a_{k} a_{l}-\bar{x}^{2}+\frac{1}{m^{2}} \sum_{k=1}^{m} \sum_{l=1}^{m} \sigma_{\mathrm{amp}_{k}} \sigma_{\mathrm{amp}_{l}} R_{k l}^{\prime}$,

where

$$
R_{k l}^{\prime}=\frac{1}{n} \sum_{j=1}^{n} x_{k j}^{\prime} x_{l j}^{\prime} .
$$

The first two terms of the right-hand side of (26) can be defined as $I$; if (3) is substituted in for $\bar{x}^{2}$, then $I=0$ and (26) can be written as 


$$
\sigma_{b}^{2}=\frac{1}{m^{2}} \sum_{k=1}^{m} \sum_{l=1}^{m} \sigma_{\mathrm{amp}_{k}} \sigma_{\mathrm{amp}_{l}} R_{k l}^{\prime}
$$

Furthermore, (28) can be rewritten as two terms:

$$
\sigma_{b}^{2}=\frac{2}{m^{2}} \sum_{k=1}^{m-1} \sum_{l=k+1}^{m} \sigma_{\mathrm{amp}_{k}} \sigma_{\mathrm{amp}_{l}} R_{k l}^{\prime}+\frac{1}{m^{2}} \sum_{k=1}^{m} \sigma_{\mathrm{amp}_{k}}^{2}
$$

because $R_{k k}^{\prime}=1$ for all $k$. Equation (29) can be further rewritten as

$$
\begin{aligned}
& \sigma_{b}^{2}=\left(\frac{m-1}{m}\right) \sigma^{2} \bar{R}^{\prime}+\frac{1}{m^{2}} \sum_{i=1}^{m} \sigma_{\mathrm{amp}_{i}}^{2}, \\
& \bar{R}^{\prime}=\frac{2}{m(m-1)} \sum_{k=1}^{m-1} \sum_{l=k+1}^{m} \frac{\sigma_{\mathrm{amp}_{k}}}{\sigma} \frac{\sigma_{\mathrm{amp}_{l}}}{\sigma} R_{k l}^{\prime} .
\end{aligned}
$$

The variance of the ensemble mean time series in Fig. $1 \mathrm{a}$ is represented by (30), whereas (31) quantifies the average value of the anomaly cross correlation coefficients (ACCC) among all EMs, weighted by average value of the variance ratio (AVR) of $\sigma_{\mathrm{amp}_{k}} \sigma_{\mathrm{amp}_{l}}$ to $\sigma^{2}$.

Substituting (30) into (5) allows $\Omega$ to be written as

$$
\Omega=\bar{R}^{\prime}+\left(\frac{1}{m-1}\right)\left(\frac{\frac{1}{m} \sum_{i=1}^{m} \sigma_{\mathrm{amp}_{i}}^{2}-\sigma^{2}}{\sigma^{2}}\right) .
$$

Here, $\Omega$ is seen to consist of two terms. Further details of the mathematical structure of $\Omega$ can now be revealed. First, note that (17) can be written as

$$
\sigma_{\mathrm{amp}_{i}}^{2}=\frac{1}{n} \sum_{j=1}^{n}\left[\left(x_{i j}-\bar{x}\right)-\left(a_{i}-\bar{x}\right)\right]^{2} .
$$

The first term on the right-hand side in (33) is

$$
\sigma_{i}^{2}=\frac{1}{n} \sum_{j=1}^{n}\left(x_{i j}-\bar{x}\right)^{2}
$$

where $\sigma_{i}^{2}$ is the variance of each EM about the mean value $\bar{x}$ in (3). The second term is

$$
\sigma_{\text {mean }_{i}}^{2}=\left(a_{i}-\bar{x}\right)^{2}
$$

where $\sigma_{\text {mean }_{i}}^{2}$ is the squared difference of the mean value $a_{i}$ of each EM to $\bar{x}$. Next, (34) and (35) are substituted into (33) to yield

$$
\sigma_{i}^{2}=\sigma_{\mathrm{amp}_{i}}^{2}+\sigma_{\operatorname{mean}_{i}}^{2}
$$

Two terms in (36) relate to the spread among EMs. One is the variance of each EM relative to its own mean, and the other relates to the difference between the mean of the EM and that across all EMs. Substitution of (36) into (32) yields

$$
\begin{aligned}
\Omega= & \left.\frac{2}{m(m-1)} \sum_{k=1}^{m-1} \sum_{l=k+1}^{m} R_{k l}^{\prime} \frac{\sigma_{\mathrm{amp}_{k}} \frac{\sigma_{\mathrm{amp}_{l}}}{\sigma}}{\sigma}\right] . \\
& +\frac{1}{m-1}\left[\frac{\frac{1}{m} \sum_{i=1}^{m}\left(\sigma_{i}^{2}-\sigma_{\text {mean }_{i}}^{2}\right)-\sigma^{2}}{\sigma^{2}}\right] .
\end{aligned}
$$

Using (4) and (34) yields

$$
\sigma^{2}=\frac{1}{m} \sum_{i=1}^{m} \sigma_{i}^{2}
$$

The above analysis leads to our final expression of $\Omega$ :

$$
\begin{aligned}
\Omega= & \frac{2}{m(m-1)} \sum_{k=1}^{m-1} \sum_{l=k+1}^{m} R_{k l}^{\prime} \frac{\sigma_{\mathrm{amp}_{k}}}{\sigma} \frac{\sigma_{\mathrm{amp}_{l}}}{\sigma} \\
& -\frac{1}{(m-1)} \frac{\frac{1}{m} \sum_{i=1}^{m} \sigma_{\text {mean }_{i}}^{2}}{\sigma^{2}}
\end{aligned}
$$

The equation is interpreted as follows. The first term on the right-hand side of (39) represents the ACCC weighted by the AVR (the "phase" and the main part of the "shape" similarity). The second term shows the similarity of the mean values among all EMs (another aspect of the "shape" similarity). The AVR is affected both by the mean values and the amplitudes of the EMs. Thus, correlation (phase) differences and shape (mean value and amplitude) differences between EMs both influence the $\Omega$ index.

\section{Clarification of the mathematical structure of $\Omega$}

The similarity among ensemble members in mediumrange forecast decreases as time increases by losing the impact of atmospheric initial conditions. At least three factors induce this decrease. One is the increase of the phase difference (hereafter, PD) among EMs (Fig. 2a). The EMs of a forecast, for example, may predict different time variations of weather conditions even though they are characterized by the same frequencies of weather change. The second factor is the mean difference (hereafter, MD) among EMs (Fig. 2b)—one EM may predict relatively warm conditions for the forecast period, whereas the other EMs may predict cooler conditions. The final factor is the amplitude difference (hereafter, AD) among EMs (Fig. 2c). For instance, large time variations of temperature may be predicted by some EMs but not in others.

In this section, we focus on how PD, MD, and AD 


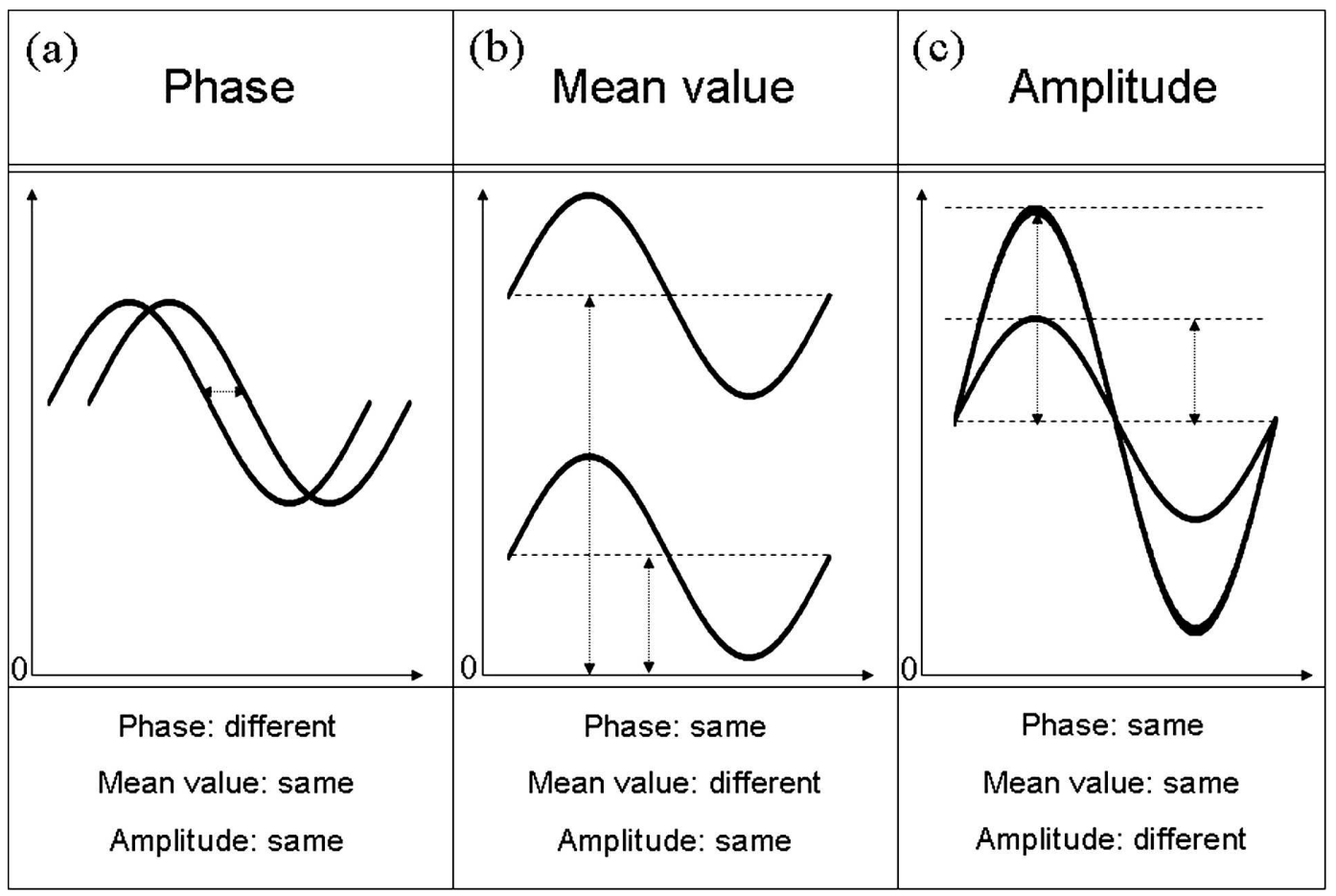

FIG. 2. Schematic diagram for similarities among EMs. It categorizes 3 types: phase, mean value, and amplitude. (a) Phase difference (PD), (b) mean difference (MD), and (c) amplitude difference (AD).

affect $\Omega$. The effect of each is isolated using hypothetical time series, as illustrated in Fig. 2.

\section{a. Influence of phase difference}

Consider a hypothetical set of EMs that has nonzero PD but zero MD and AD, as shown in Fig. 3. The time series in (40) is a sine curve with $m$ EMs; here, $n$ is the

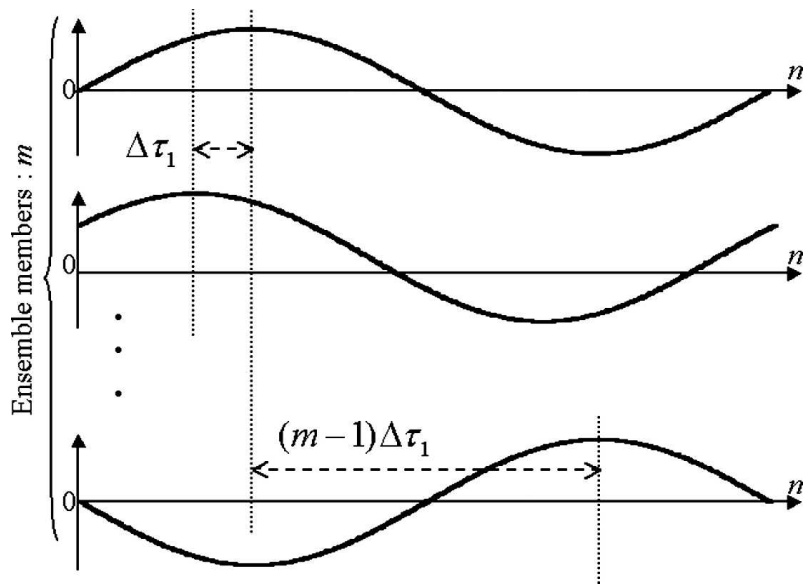

FIG. 3. Schematic figure of EMs in a hypothetical case. All EMs consist of sine curves, which have $\operatorname{PD}\left(\Delta \tau_{1}\right)$. There is no MD or AD among EMs. total number of time periods (wavelength), $j$ is the specific time period, $i$ is the number of EMs $(m=2-16$ for every two steps), and $\Delta \tau_{1}$ is the PD in each $\operatorname{EM}(0-2 \pi)$ :

$$
x_{i j}=\sin \left[\frac{2 \pi j}{n}-(i-1) \Delta \tau_{1}\right] .
$$

When (40) is substituted into matrix $\mathbf{A}$, we produce the functional relationship between $\Omega$ and $\Delta \tau_{1}$ shown in

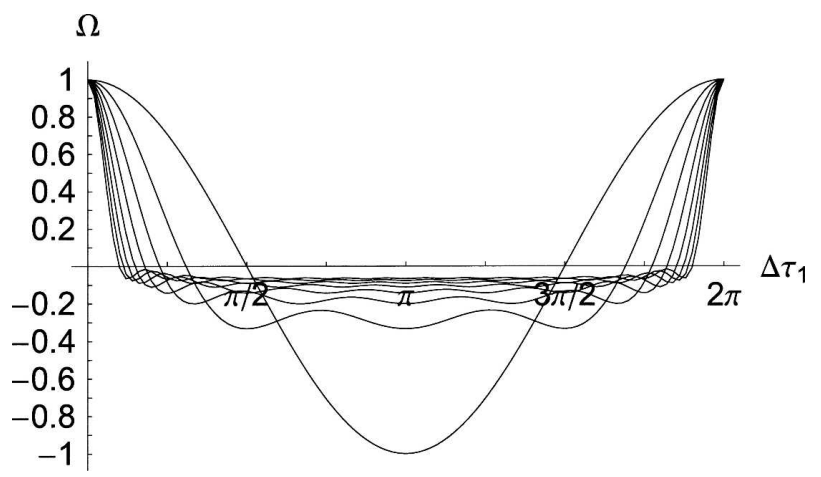

FIG. 4. Behavior of $\Omega$ in response to $\operatorname{PD}\left(\Delta \tau_{1}\right)$ in case of different sets of EMs ( $m=2-16$ for every two steps). The abscissa $\left(\Delta \tau_{1}\right)$ is PD among each EM. Time periods $(n)$ are 100 . When $\Delta \tau_{1}$ equals $\pi, \Omega$ approximates 0 with large sets of EMs; $\Omega$ goes between approximately 0 and 1 when there are a large number of EMs (in this case, $m$ is 16 ). When $m$ equals $2, \Omega$ goes between -1 and 1 . 


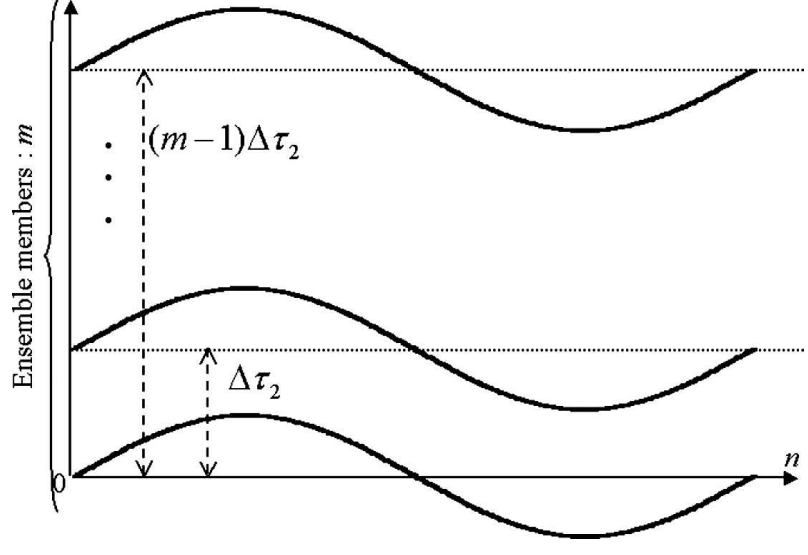

FIG. 5. Schematic figure of EMs in a hypothetical case. All EMs consist of sine curves, which have $\operatorname{MD}\left(\Delta \tau_{2}\right)$. There is no PD or $\mathrm{AD}$ among EMs.

Fig. 4. The second term of the right-hand side of (39) vanishes because the MD for the EMs is zero. The AD among all EMs is also zero for this idealized case:

$$
\sum_{k=1}^{m-1} \sum_{l=k+1}^{m} \frac{\sigma_{\mathrm{amp}_{k}}}{\sigma} \frac{\sigma_{\mathrm{amp}_{l}}}{\sigma}=1 .
$$

Substituting (41) into (39) yields

$$
\Omega=\frac{2}{m(m-1)} \sum_{k=1}^{m-1} \sum_{l=k+1}^{m} R_{k l}^{\prime},
$$

which shows how the degree of PD among the EMs affects $\Omega$. Figure 4 shows how $\Omega$ varies with PD for each $m$ considered; $\Omega$ is small for large sets of EMs when $\Delta \tau_{1}$ equals $\pi$. If there is no PD (PD $=0,2 \pi$ ) among EMs, $\Omega$ is 1 regardless of the number of EMs. When $m$ is 2,

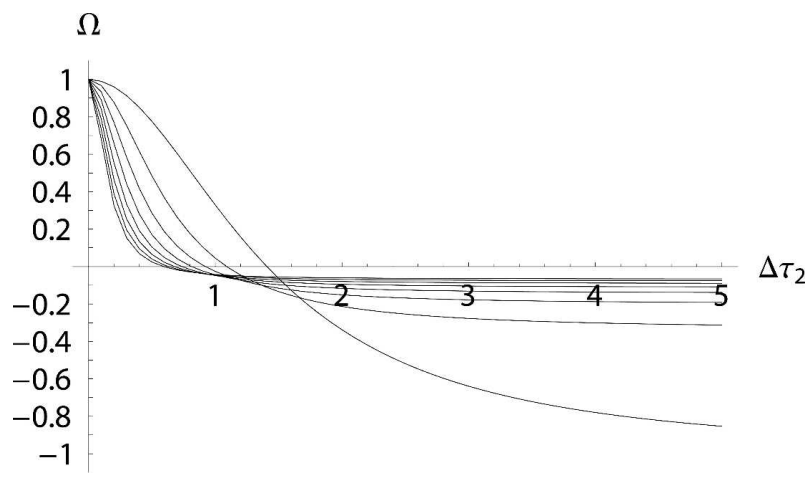

FIG. 6. Behavior of $\Omega$ in response to mean value difference (MD). The abscissa denotes MD $\left(\Delta \tau_{2}\right)$. When $\Delta \tau_{2}$ is large (e.g., $\left.\Delta \tau_{2}=5\right), \Omega$ varies to approximately 0 with large sets of EMs. $\Omega$ goes between approximately 0 and 1 when there are fully number of EMs ( $m=16$ in this case). When $m$ equals $2, \Omega$ shows between -1 and 1 .

\section{Second term of $\Omega$}

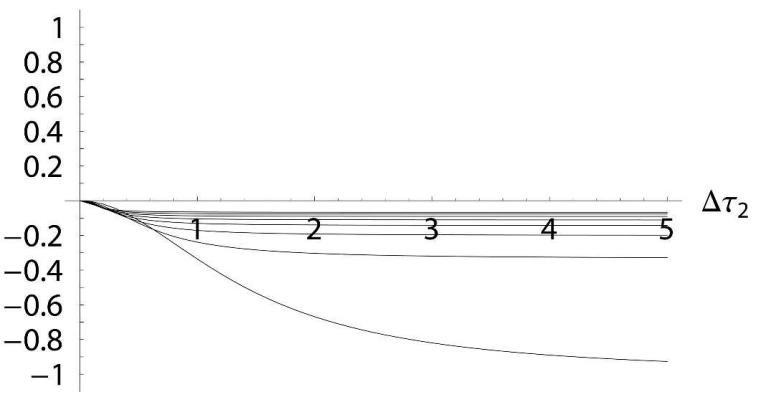

FIG. 7. Response of the second term of the right-hand side of $\Omega$ in Eq. (45) to $\operatorname{MD}\left(\Delta \tau_{2}\right)$. When $\Delta \tau_{2}$ is large (e.g., $\Delta \tau_{2}=5$ ), $\Omega$ approximates 0 with large sets of EMs. In case of $m=2$, the second term varies between -1 and approximately 0 . Therefore, this term cannot be negligible. On the other hand, in case of $\mathrm{m}=$ 16 , the second term is negligible for its small value.

$\Omega$ behaves like a cross correlation coefficient; if it is correlated (uncorrelated), then $\Omega$ approaches $1(-1)$. In contrast, when the number of EMs is large enough (or, in this case, when $m=16$ ), $\Omega$ is effectively 0 for all nonzero PD. The value of $\Omega$ is not identically 0 when $\mathrm{PD}=\pi$ because in this case, $\sigma_{b}^{2}$ becomes 0 , and (5) can be expressed as

$$
\Omega=-\frac{1}{m-1} .
$$

In this case only, (42) cannot explain $\Omega$. As a result, Eq. (42), which measures the phase similarity (correlation), varies between 0 and 1 for the case of infinite EMs. This result shows that $\Omega$ can approach 0 from the PD alone.

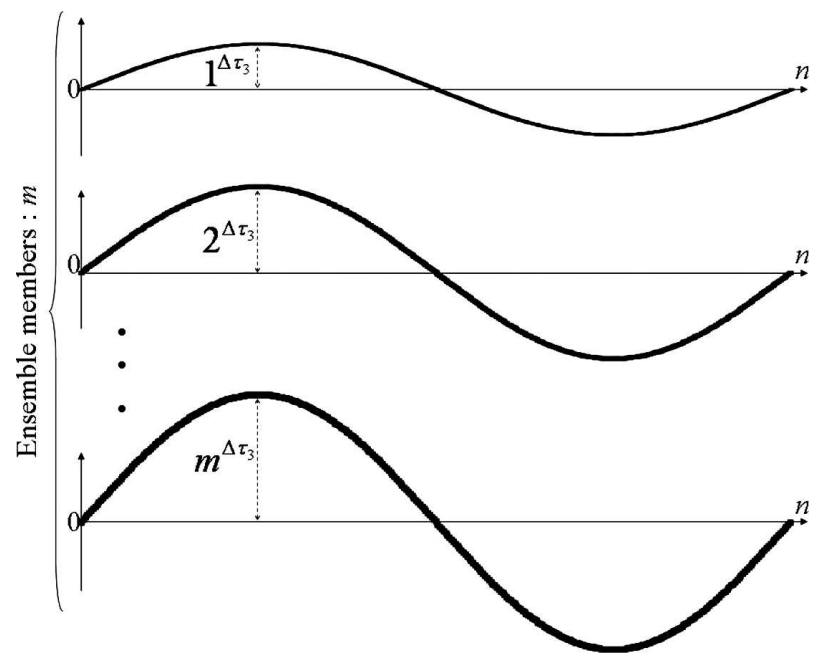

FIG. 8. Schematic figure of EMs in a hypothetical case. Every EM consists of sine curve with different amplitude. Every EM has $\operatorname{AD}\left(\Delta \tau_{3}\right)$. There is no PD or MD among EMs. 


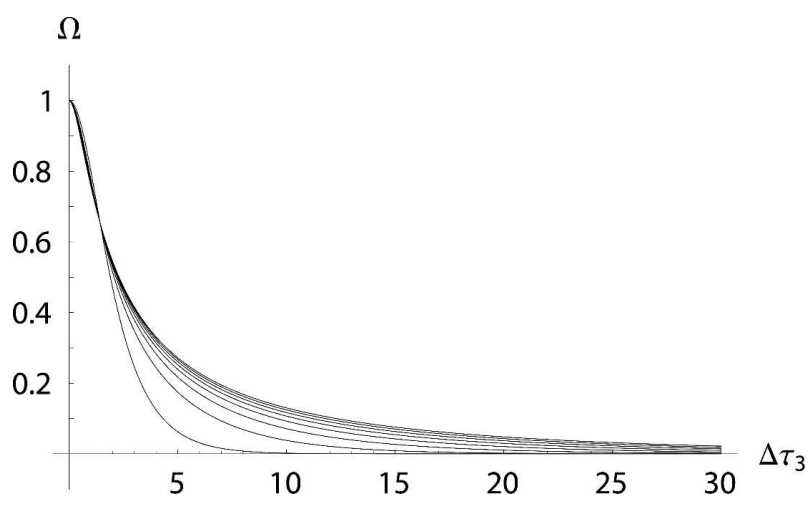

FIG. 9. Behavior of $\Omega$ in response to amplitude difference (AD). The abscissa denotes $\mathrm{AD}\left(\Delta \tau_{3}\right)$. When $\Delta \tau_{3}$ equals $5, \Omega$ indicates a large value with a large number of EMs. In case of $\Delta \tau_{3}=0$, all EMs show identical amplitude and $\Omega$ becomes $1 . \Omega$ varies between 0 and 1 irrespective of number of EMs.

\section{b. Influence of mean value difference}

Consider the behavior of $\Omega$ when MD is nonzero but there is no PD or AD, as shown in Fig. 5. The first term on the right-hand side of (44) is the mean value of each $\mathrm{EM}$, and the second term is a simple sine curve:

$$
x_{i j}=(i-1) \Delta \tau_{2}+\sin \frac{2 \pi j}{n} .
$$

Furthermore, $i$ is the index of the ensemble member, $j$ is the time period number, $n$ is the total number of time periods, and $\Delta \tau_{2}$ is an index of MD among EMs. There is no PD among EMs in this hypothetical, idealized case. Therefore, (39) can be written as

$$
\begin{aligned}
\Omega= & \frac{2}{m(m-1)} \sum_{k=1}^{m-1} \sum_{l=k+1}^{m} \frac{\sigma_{\mathrm{amp}_{k}}}{\sigma} \frac{\sigma_{\mathrm{amp}_{l}}}{\sigma} \\
& -\frac{1}{m-1} \frac{\frac{1}{m} \sum_{i=1}^{m} \sigma_{\text {mean }_{i}}^{2}}{\sigma^{2}} .
\end{aligned}
$$

Substitution of (44) into matrix A yields the $\Omega$ values shown in Fig. 6; the figure plots $\Omega$ versus $\operatorname{MD}\left(\Delta \tau_{2}\right)$ for several ensemble sizes $(m=2-16)$. Figure 7 plots the second term on the right-hand side of (45) versus $\Delta \tau_{2}$. In both figures, the values approach 0 for large sets of EMs when $\Delta \tau_{2}$ is large (e.g., $\Delta \tau_{2}=5$ ). That is, the impact of the second term on the right-hand side of (45) is negligible in the case of a large number of EMs, for which the $\Omega$ equation simplifies to

$$
\Omega \cong \frac{2}{m(m-1)} \sum_{k=1}^{m-1} \sum_{l=k+1}^{m} \frac{\sigma_{\mathrm{amp}_{k}}}{\sigma} \frac{\sigma_{\mathrm{amp}_{l}}}{\sigma} .
$$

When there is no MD between EMs, the second term of the right-hand side is 0 , and $\Omega$ is 1 , regardless of $m$.

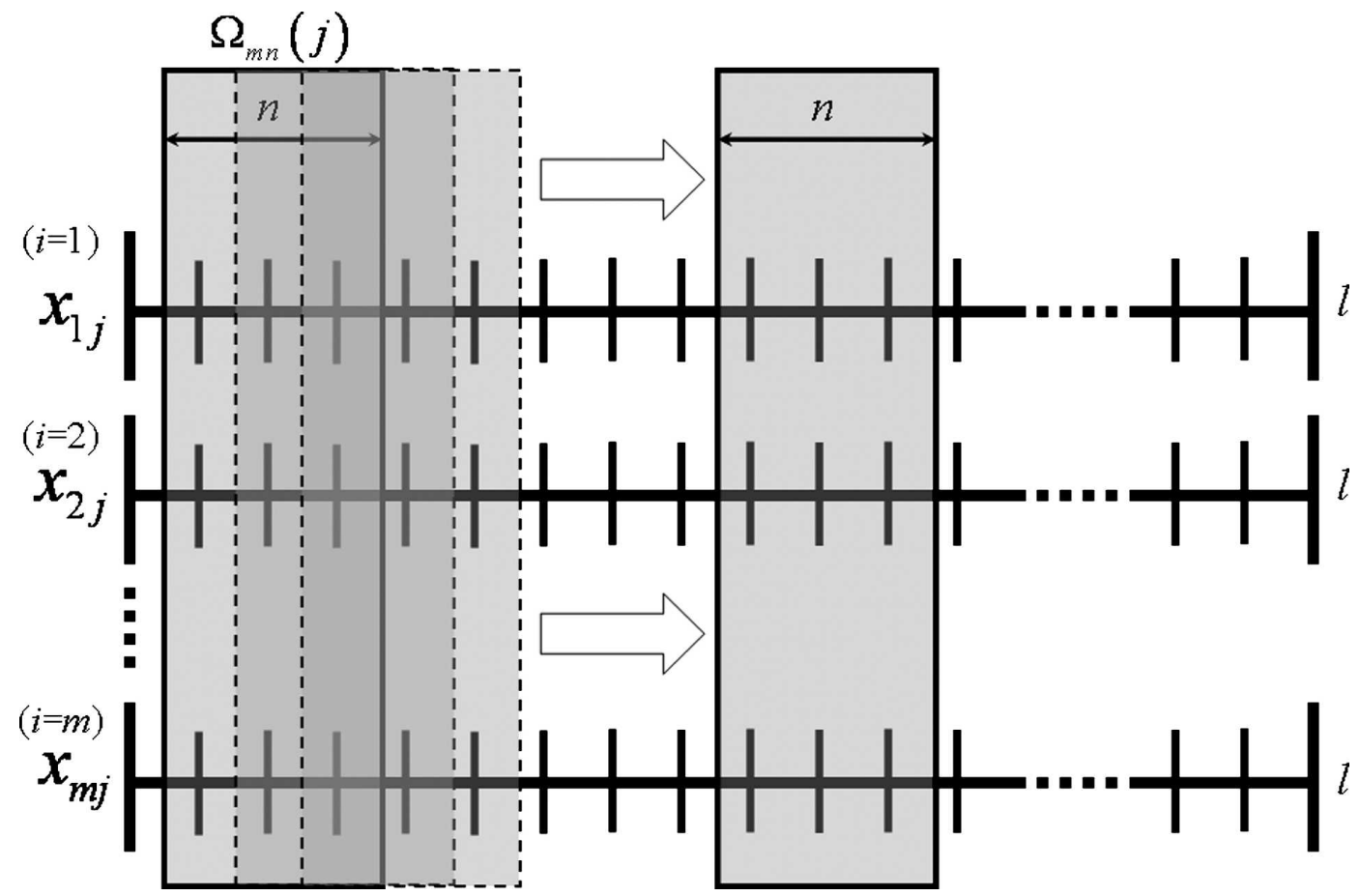

FIG. 10. Schematic figure of $\Omega_{m n}(j)$. Here $\Omega_{m n}(j)$ is estimated at every time step $(j)$. Here $l$ : total number of time periods, $m$ : ensemble members, and $n$ : time periods to estimate $\Omega_{m n}(j)$. 
500hPa, Dec., 16 ensemble members, $46 \mathrm{~N}, 180 \mathrm{E}$

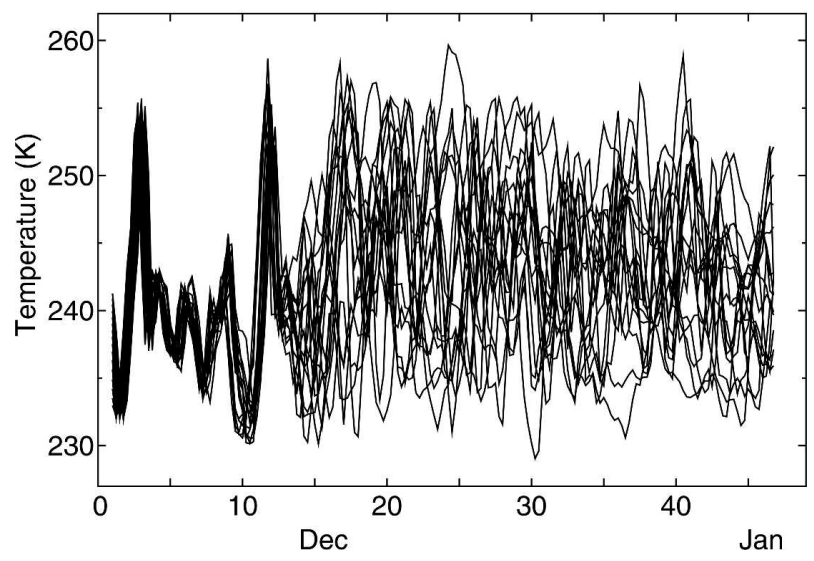

FIG. 11. Time series of $16 \mathrm{EMs}$ of temperature at 500-hPa height over a grid cell in December $\left(46^{\circ} \mathrm{N}, 180^{\circ}\right)$.

When $m=2, \Omega$ approaches -1 as the MD increases. If there are many EMs ( $m=16$ in this experiment), $\Omega$ vanishes with increasing $\mathrm{MD}$.

\section{c. Influence of amplitude difference}

Now consider a hypothetical time series of EMs, as in (47), in which $\mathrm{AD}$ is nonzero but $\mathrm{PD}$ and $\mathrm{MD}$ are zero ( $m=2-16$ for every two steps). Figure 8 shows a schematic of (47):

$$
x_{i j}=i^{\Delta \tau_{3}} \sin \frac{2 \pi j}{n} .
$$

$\Omega$ vs. ACCC vs. AVR

500hPa, Dec., 16 ensemble members, 46N, 180E

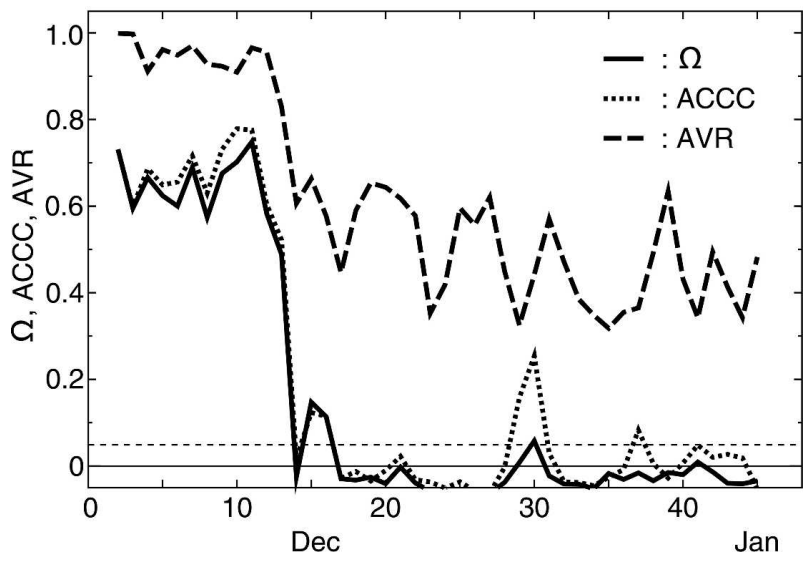

FIG. 12. Time series of $\Omega_{m n}(j), \operatorname{ACCC}_{m n}(j)$, and $\operatorname{AVR}_{m n}(j)$ of temperature at 500 -hPa height over a grid cell in December $\left(46^{\circ} \mathrm{N}, 180^{\circ}\right)$. Time period is 3 days $(n=12)$ for every line. Short dashed line represents the $92 \%$ significance level.

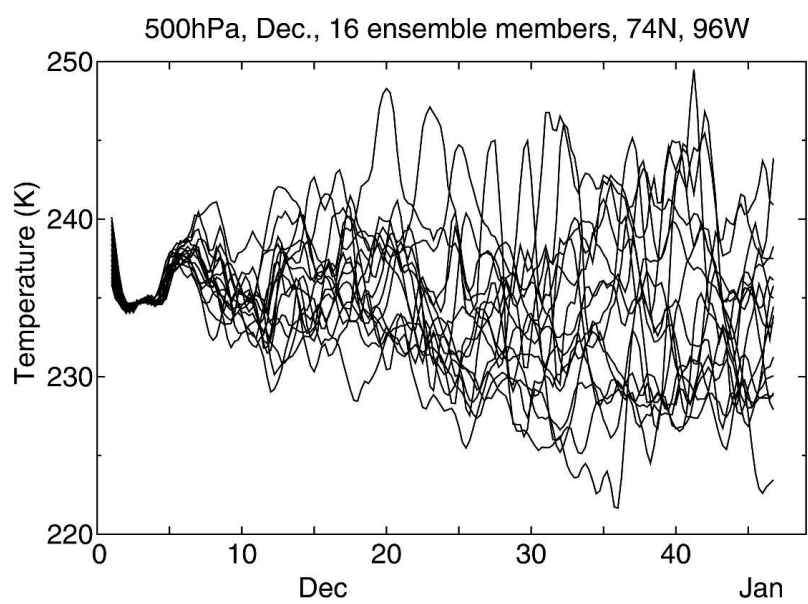

FIG. 13. Time series of $16 \mathrm{EMs}$ of temperature at 500-hPa height over a grid cell in December $\left(74^{\circ} \mathrm{N}, 96^{\circ} \mathrm{W}\right)$.

The time series in (47) is a sine curve with $m$ EMs; here, $\Delta \tau_{3}$ is an index that shows the AD among EMs. In this hypothetical case, (39) can be expressed as

$$
\Omega=\frac{2}{m(m-1)} \sum_{k=1}^{m-1} \sum_{l=k+1}^{m} \frac{\sigma_{\mathrm{amp}_{k}}}{\sigma} \frac{\sigma_{\mathrm{amp}_{l}}}{\sigma} .
$$

Figure 9 plots $\Omega$ versus AD $\left(\Delta \tau_{3}\right)$ for several EMs $(m=$ 2-16 for every two steps). The figure shows that when $\Delta \tau_{3}$ is moderately small (e.g., $\Delta \tau_{3}=5$ ), $\Omega$ increases with increasing ensemble size. In contrast, $\Omega$ approaches 0 when the amplitudes are quite different amongst the EMs, despite the zero values of PD and MD. The $\Omega$

$\Omega$ vs. ACCC vs. AVR

$500 \mathrm{hPa}$, Dec., 16 ensemble members, 74N, 96W

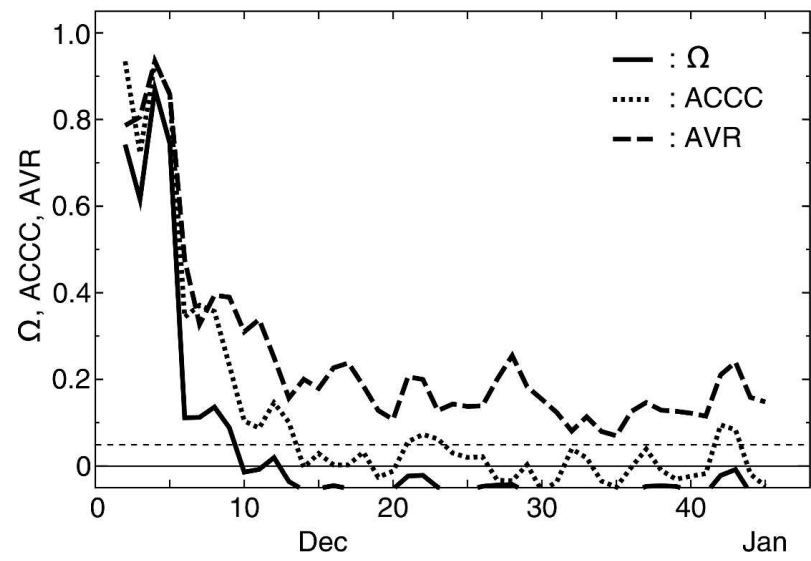

FIG. 14. Time series of $\Omega_{m n}(j), \operatorname{ACCC}_{m n}(j)$, and $\operatorname{AVR}_{m n}(j)$ of temperature at $500-\mathrm{hPa}$ height over a grid cell in December $\left(74^{\circ} \mathrm{N}, 96^{\circ} \mathrm{W}\right)$. Time period is 3 days $(n=12)$ for every line. Short dashed line represents $92 \%$ significance level. 
500hPa, Dec., 16 ensemble members, $\mathrm{n}: 12$ (3days)
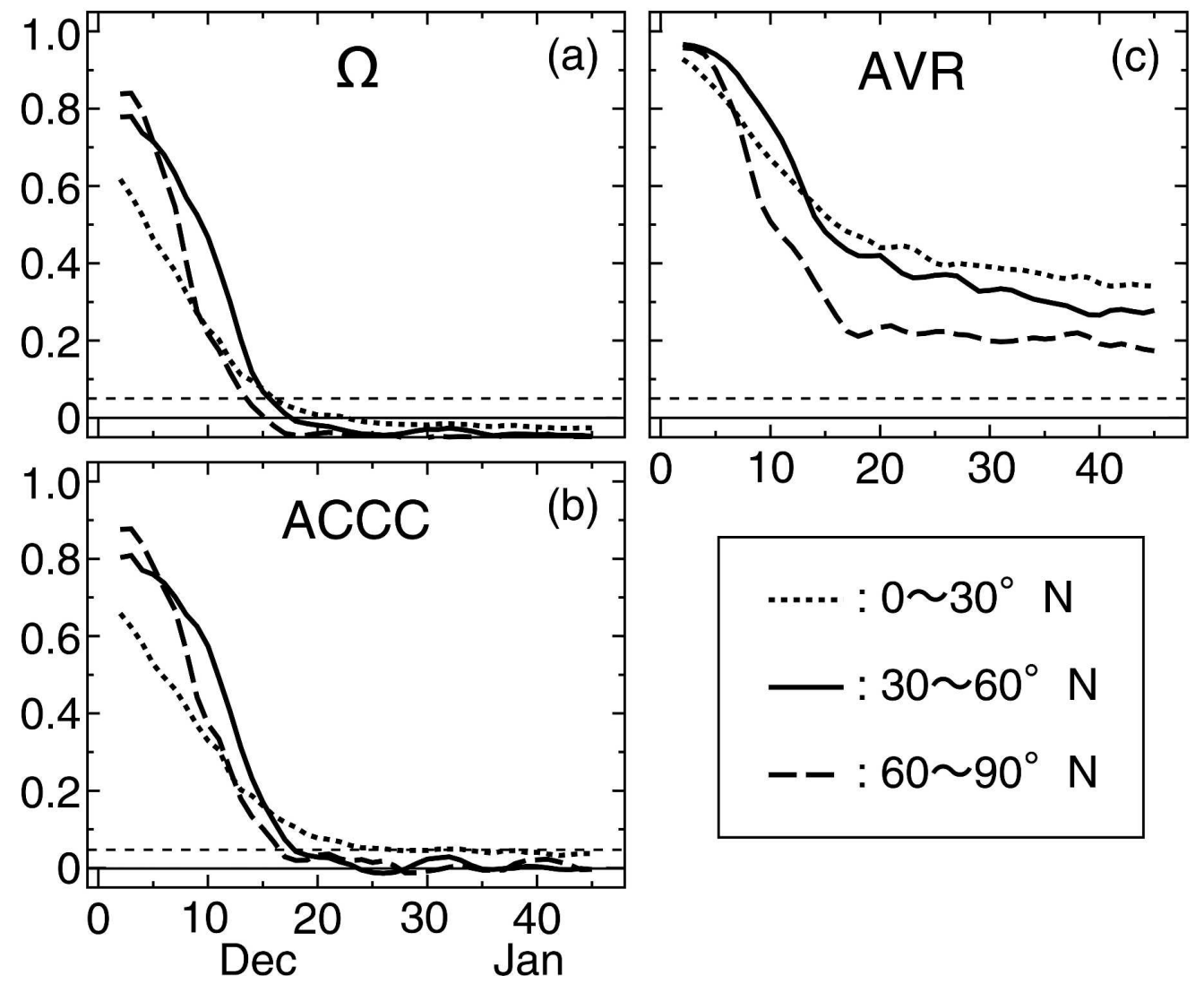

FIG. 15. Time series of $\Omega_{m n}(j), \operatorname{ACCC}_{m n}(j)$, and $\operatorname{AVR}_{m n}(j)$ of temperature at 500-hPa height averaged over low $\left(0^{\circ}-30^{\circ} \mathrm{N}\right)$, middle $\left(30^{\circ}-60^{\circ} \mathrm{N}\right)$, and high $\left(60^{\circ}-90^{\circ} \mathrm{N}\right)$ latitudes in December. Time period is 3 days $(n=12)$ for every line.

values are constrained to lie between 0 and 1 when $\mathrm{AD}$ acts alone.

\section{d. Mathematical characteristics of three indices}

Two indices clarify the impact of PD and the joint impact of $\mathrm{MD}$ and $\mathrm{AD}$ on the behavior of $\Omega$. First, (42) provides the average value of the ACCC. The ACCC shows the impact of PD on $\Omega$, regardless of MD and AD. A second index, defined in (46), is the average value of the AVR. The AVR indicates the impact of $\mathrm{MD}$ and $\mathrm{AD}$ on $\Omega$, regardless of PD. Here we assume that there is a large number of EMs and the impact of the second term in (45) can be neglected.

Note that while the value of $\Omega$ reflects the values of both ACCC and AVR, there is no simple functional form relating the three quantities. The ACCC and AVR are simply presented as the "phase" and "shape" aspects of $\Omega$.

To summarize the results of the present section, we note that two types of statistical differences amongst ensemble members underlie $\Omega$ : phase differences (PD, as characterized by the ACCC), and shape differences (MD and $\mathrm{AD}$, as characterized by the AVR). PD, MD, and $\mathrm{AD}$ affect the value of $\Omega$ in different ways, as illustrated in Figs. 4, 6, and 9. The concepts of ACCC and AVR will be used extensively in the next section.

\section{Predictability among ensemble members in medium-range forecast using the new estimation methods}

In this section, we use the mathematical structure of $\Omega$ revealed above to evaluate the predictability among ensemble members in medium-range forecast from a viewpoint of similarity. Here the predictability is defined as the impact of initial conditions on atmospheric behavior. We deal with idealized predictability; we do not compare the model ensemble results with observations. In essence, we assume that the model we use is perfect and that one of the EMs represents "nature"- 
that is, it shows the true evolution of the various atmospheric fields.

\section{a. Model and data}

Ensemble numerical simulations were integrated with the Center for Climate System Research (CCSR; University of Tokyo) and the National Institute for Environmental Studies (CCSR/NIES) AGCM (Numaguti et al. 1997). The CCSR/NIES model used T42 horizontal truncation $\left(128 \times 64\right.$ grid cells, approximately $2.8^{\circ}$ resolution) and 20 sigma coordinate layers in the vertical.

\section{b. Methodology}

Similarity among ensemble members in mediumrange forecast was determined with (49), in which $j$ is the elapsed time (h), $n$ is the number of periods evaluated at every time step, and $m$ is the number of EMs; $\Omega_{m n}(j)$ was calculated for all $j$, as shown in Fig. 10:

$$
\Omega_{m n}(j)=\frac{m \sigma_{b}^{2}(j)-\sigma^{2}(j)}{(m-1) \sigma^{2}(j)} .
$$

The ACCC and AVR for such an analysis can be computed with

$$
\begin{aligned}
\operatorname{ACCC}_{m n}(j) & =\frac{2}{m(m-1)} \sum_{k=1}^{m-1} \sum_{l=k+1}^{m} R_{k l}^{\prime}(j) \\
\operatorname{AVR}_{m n}(j) & =\frac{2}{m(m-1)} \sum_{k=1}^{m-1} \sum_{l=k+1}^{m} \frac{\sigma_{\mathrm{amp}_{k}}(j)}{\sigma(j)} \frac{\sigma_{\mathrm{amp}_{l}}(j)}{\sigma(j)}
\end{aligned}
$$

Again, $\operatorname{ACCC}_{m n}(j)$ indicates the "phase predictability" and $\operatorname{AVR}_{m n}(j)$ the "shape predictability" among ensemble members. As the single statistical quantity that includes both phase and shape predictability, $\Omega_{m n}(j)$ indicates "similarity predictability."

\section{c. Grid scale}

Figure 11 shows the time series of temperature at 500 hPa produced by $16 \mathrm{EMs}(m=16)$ at a specific grid cell $\left(46^{\circ} \mathrm{N}, 180^{\circ}\right)$ in December. The 16 sets of 1 December atmospheric initial conditions used were constructed with the 1-h lagged approach. All data in Fig. 11 are averaged over $6 \mathrm{~h}$. Figure 12 shows time series of $\Omega_{m n}(j), \operatorname{ACCC}_{m n}(j)$, and $\operatorname{AVR}_{m n}(j)$ as calculated from the 16 EMs. Every point of each line was calculated from 3 days of data $(n=12)$. Here $\operatorname{ACCC}_{m n}(j)$ was approximately constant (and thus showing stable phase predictability) at 0.7 until day 12 , and $\operatorname{AVR}_{m n}(j)$ was nearly constant around 0.9 until day 12 ; thus, large predictability for shape also persisted. Large predictability
$\Omega$ vs. ACCC vs. AVR

$500 \mathrm{hPa}$, Dec., 16 ensemble members, Global Mean

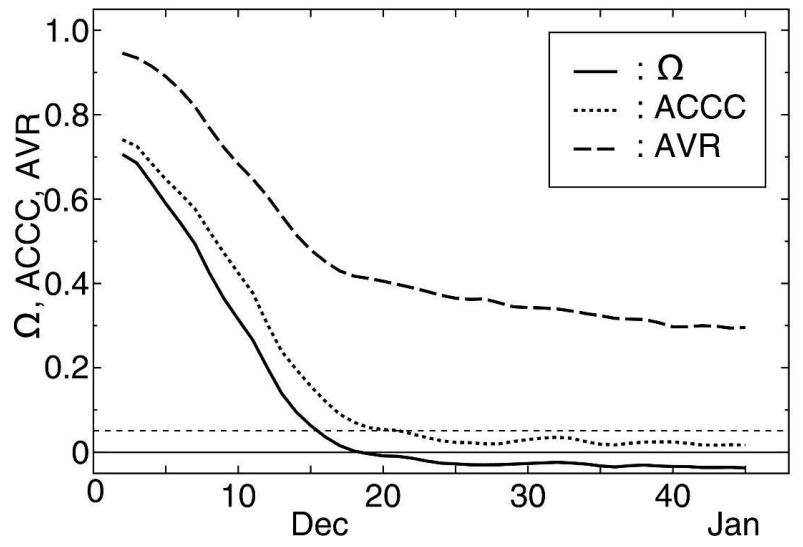

FIG. 16. Time series of $\Omega_{m n}(j), \operatorname{ACCC}_{m n}(j)$, and $\operatorname{AVR}_{m n}(j)$ of temperature at $500-\mathrm{hPa}$ height averaged over globe in December. Time period is 3 days $(n=12)$ for every line.

for both phase and shape was equivalent to a large value of $\Omega_{m n}(j)$.

After day $12, \Omega_{m n}(j)$ and $\operatorname{ACCC}_{m n}(j)$ decreased to values less than 0.05 , a decrease significant at the $92 \%$ level, according to Monte Carlo analysis; $\operatorname{AVR}_{m n}(j)$ also decreased at this time, but the decrease was much smaller than that for $\operatorname{ACCC}_{m n}(j)$. The impact of $\operatorname{ACCC}_{m n}(j)$ on $\Omega_{m n}(j)$ was therefore dominant. After losing shape predictability around day $14, \operatorname{AVR}_{m n}(j)$ fluctuated between 0.3 and 0.6. By this time, the impact of the initial conditions on the forecast had disappeared.

Note that physical constraints on meteorological fields limit the degree to which $\operatorname{AVR}_{m n}(j)$ can be reduced. In both the real world and models, temperatures, for example, will not vary by the amounts needed to reduce $\operatorname{AVR}_{m n}(j)$ to 0 . Thus, our use of the term "shape predictability" throughout this text when referring to $\operatorname{AVR}_{m n}(j)$, though convenient, is not rigorously correct; in the strictest sense, "shape predictability" would refer to the degree to which the $\operatorname{AVR}_{m n}(j)$ exceeds the lower limit. In any case, for the grid cell examined in Fig. 11 and 12, large decreases in both $\operatorname{ACCC}_{m n}(j)$ and $\operatorname{AVR}_{m n}(j)$ induce the large decrease in $\Omega_{m n}(j)$ at day 12 - chaos destroys both the phase and shape similarity at approximately the same time.

Figure 13 presents the time series of $500-\mathrm{hPa}$ temperature at a different grid cell $\left(74^{\circ} \mathrm{N}, 96^{\circ} \mathrm{W}\right)$. As shown in Fig. 14, the corresponding $\Omega_{m n}(j)$ and $\operatorname{ACCC}_{m n}(j)$ values show large decreases after day 5 . The decease in $\Omega_{m n}(j)$ is in fact significantly larger than that in $\operatorname{ACCC}_{\mathrm{mn}}(j)$ due to a sharp decrease in $\operatorname{AVR}_{m n}(j)$. At high latitudes, SST has a small impact on atmospheric 

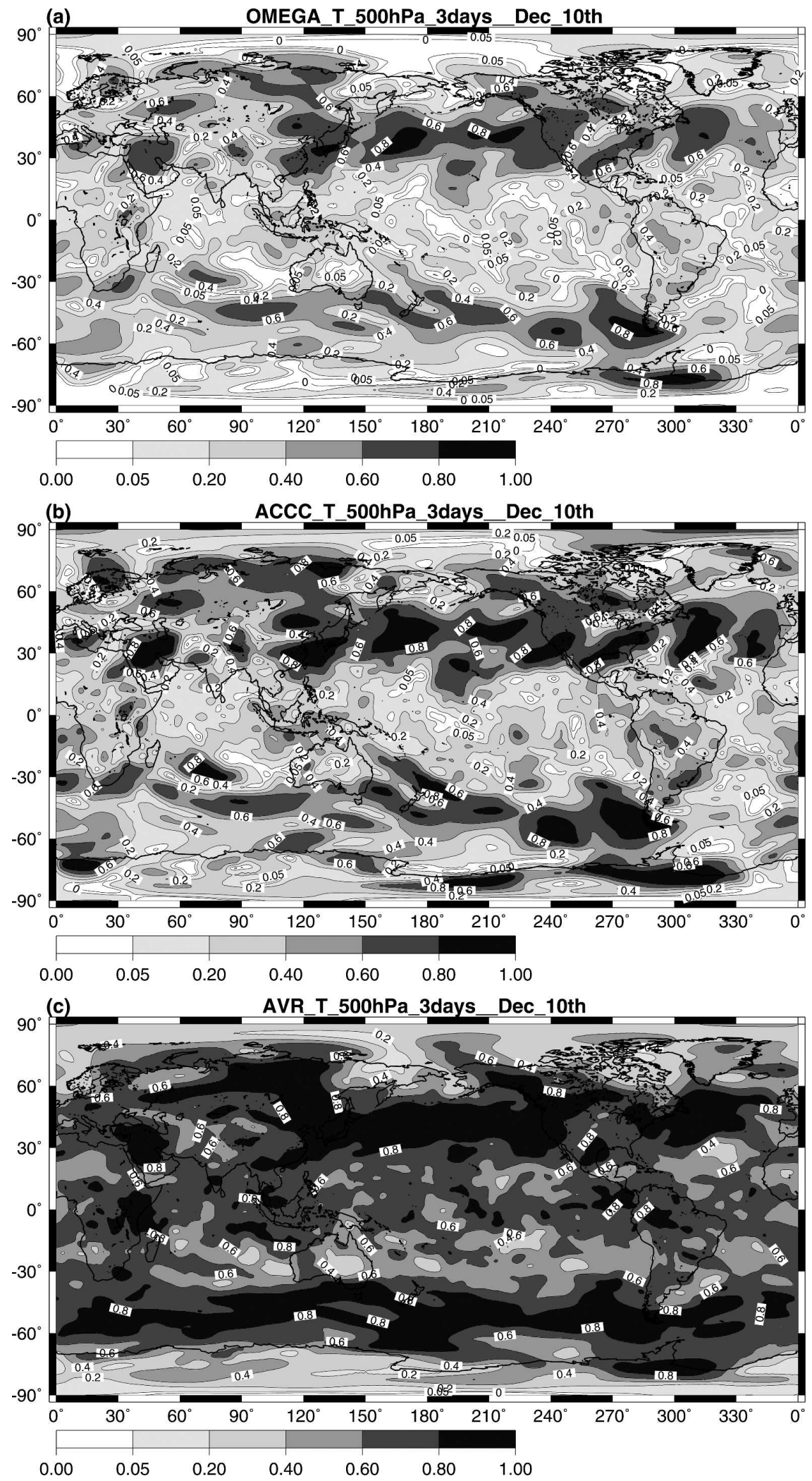

Fig. 17. Global distributions of (a) $\Omega_{m n}(j)$, (b) $\operatorname{ACCC}_{m n}(j)$, and (c) $\operatorname{AVR}_{m n}(j)$ of temperature at $500-\mathrm{hPa}$ height on 10 December All figures are calculated with 12 time periods (3 days). 
behavior, especially during winter. This may explain the sharp decrease in $\operatorname{AVR}_{m n}(j)$. Here, predictability decreased not only because of an increase in PD but also due to increases in MD and AD.

\section{d. Zonal mean}

Figure 15 shows (a) $\Omega_{m n}(j)$, (b) $\operatorname{ACCC}_{m n}(j)$, and (c) $\mathrm{AVR}_{m n}(j)$ for 500-hPa temperature at low, middle, and high latitudes. In all figures, dotted, solid, and dashed lines indicate zonal averages between $0^{\circ}$ and $30^{\circ} \mathrm{N}$, between $30^{\circ}$ and $60^{\circ} \mathrm{N}$, and between $60^{\circ}$ and $90^{\circ} \mathrm{N}$, respectively.

Consider the behavior of $\mathrm{ACCC}_{m n}(j)$ in Fig. 15b. On day 2 , the high-latitude average value is larger than that at other latitudes. However, phase predictability is lost first at high latitudes, with values decreasing to 0.05 on day 16. In contrast, low-latitude values are initially about 0.65 , but the phase predictability there persists until day 24. Midlatitude phase predictability shows the largest value from day 7 to 15 among the three latitudinal bands and lasts until day 17.

Figure $15 \mathrm{c}$ shows $\operatorname{AVR}_{m n}(j)$ for the three latitudinal bands. All three lines show similar values in the first few days, implying that shape predictability at early times has no latitudinal dependence; $\operatorname{AVR}_{m n}(j)$ at high latitudes, however, decreases first and then stabilizes after day 16. At mid- and low latitudes, the values take much longer to decrease. Comparison of Fig. 15b and $15 \mathrm{c}$ shows that phase and shape predictability vanish at high latitudes simultaneously. In lower and midlatitudes, however, shape predictability persists for several days after phase predictability is lost. Thus, at these latitudes, the effects of atmospheric chaos differ for phase and shape.

Figure 15a shows $\Omega_{m n}(j)$, the measure of comprehensive predictability, as a function of time at low, middle, and high latitudes. The relative positions of the lines in Fig. 15a look very similar to those for $\operatorname{ACCC}_{m n}(j)$ in Fig. 15b. At all three latitudes, however, note that $\Omega_{m n}(j)$ reached 0.05 between days 13 and 16 , somewhat earlier than did $\mathrm{ACCC}_{m n}(j)$.

\section{e. Global mean}

Figure 16 shows $\Omega_{m n}(j), \operatorname{ACCC}_{m n}(j)$, and $\operatorname{AVR}_{m n}(j)$ for $500-\mathrm{hPa}$ temperature averaged worldwide in December. Here $\Omega_{m n}(j)$ decreases with time and reaches 0.05 on day 15 . Therefore, by this measure, predictability has a time scale of 15 days. Predictability for $\operatorname{ACCC}_{m n}(j)$, however, persists for more than 5 days longer. After a sharp decrease, $\operatorname{AVR}_{m n}(j)$ shows stable values of about 0.3 starting at day 20. Figure 16 shows nearly constant differences between $\Omega_{m n}(j)$ and
$\operatorname{ACCC}_{m n}(j)$ at every time period, suggesting that the impact of MD and AD on predictability at global scales does not vary with time.

\section{f. Global distribution}

Figure 17 shows global distributions of $\Omega_{m n}(j)$, $\operatorname{ACCC}_{m n}(j)$, and $\operatorname{AVR}_{m n}(j)$ for 500-hPa temperature on day 10. Values are calculated with 12 time periods from day 9 to 11. Large values of both $\operatorname{ACCC}_{m n}(j)$ and $\operatorname{AVR}_{m n}(j)$ occur over midlatitudes, especially over the oceans. The regional distributions resemble strong westerly jets in which long-period waves dominate atmospheric behavior. The large values of $\operatorname{ACCC}_{m n}(j)$ and $\operatorname{AVR}_{m n}(j)$ in certain regions lead to correspondingly large values of $\Omega_{m n}(j)$ in these regions. Predictability can be maintained not only for PD but also for MD and $\mathrm{AD}$ in midlatitudes.

At high latitudes, however, $\operatorname{ACCC}_{m n}(j)$ and $\operatorname{AVR}_{m n}(j)$ are small, and thus $\Omega_{m n}(j)$ is also small. At low latitudes, relatively large values of $\operatorname{AVR}_{m n}(j)$ persist on day 10. In contrast, small values of $\operatorname{ACCC}_{m n}(j)$ occurred over many tropical regions, and $\Omega_{m n}(j)$ is small there, with values between 0 and 0.2 . Thus, in the Tropics, similarity predictability is lost due to PD among EMs. The factors that reduce predictability are seen to have a latitudinal dependence.

\section{Summary}

Two interpretations of the mathematical structure of the statistical similarity index $\Omega$ are provided. The first interpretation shows that, under the assumption that both boundary forcing and atmospheric chaos contribute separately to the value of a meteorological variable at a given time step, $\Omega$ is equivalent to the square of the correlation coefficient between the variable and the forcing. The nature of this boundary forcing, which presumably is multivariate and multifaceted, need not be established or understood for the calculation of $\Omega$.

The second interpretation, the mathematically stricter one, shows $\Omega$ to be associated with two quantities: the average value of the anomaly cross correlation coefficient (ACCC) and the average value of the variance ratio (AVR) amongst the EMs. The second term indicates one part of the similarity of mean values amongst the EMs, but this term could be negligible for large numbers of EMs, and thus the first term dominates the behavior of $\Omega$. The statistical characteristics of $\Omega$ suggest that $\Omega$ reflects both phase similarity (correlation) and shape similarity (mean value and amplitude). It thus has an advantage over both the cross correlation coefficient, which shows similarity of phase 
but not shape among EMs, and the root-mean-square (RMS) difference, which indicates similarities in shape including the effect of period characteristics.

Skill in ensemble weather forecasts is typically estimated with anomaly correlations or RMS differences. Even if large predictability for shape is estimated with the RMS difference, such skill may not be practical or reliable in the face of small predictability for phase similarity. The converse is also true. This paper suggests that by characterizing phase and shape predictability jointly, the $\Omega_{m n}(j)$ diagnostic may be a superior predictability measure. In addition, this study shows that relative losses (with time) in phase and shape predictability vary with latitude. As we described in section 5, this study does not deal with real predictability but idealized predictability by assuming that the model is perfect and that one of the EMs represents "nature." However, it may be mathematically possible to estimate the real predictability with $\Omega_{m n}(j)$ by calculating the anomalies of each EM compared with observation data. The predictability depends on which time scale we focus on; $\Omega_{m n}(j)$ can be evaluated for any averaging time scale, and this can be one of the suitable characteristics to estimate the predictability. Overall, $\Omega_{m n}(j)$ is seen to be a highly practical and versatile tool for the analysis of ensemble weather forecasts and perhaps for other scientific and technological applications as well.

Acknowledgments. The first author is funded by the Japan Society for the Promotion of Science (JSPS) as a post-doctoral research fellow. We are grateful to Dr. Masao Kanamitsu for helpful discussions and comments on the work presented herein. All computational resources were provided by the National Institute for Environmental Studies.

\section{REFERENCES}

Dirmeyer, P. A., R. D. Koster, and Z. Guo, 2006: Do global models properly represent the feedback between land and atmosphere? J. Hydrometeor., 7, 1177-1198.

Epstein, E. S., 1969: Stochastic dynamic prediction. Tellus, 21, 739-759.

Guo, Z., and Coauthors, 2006: GLACE: The Global LandAtmosphere Coupling Experiment. Part II: Analysis. J. Hydrometeor., 7, 611-625.

Kimoto, M., H. Mukougawa, and S. Yoden, 1992: Medium-range forecast skill variation and blocking transition: A case study. Mon. Wea. Rev., 120, 1616-1627.
Koster, R. D., M. J. Suarez, and M. Heiser, 2000: Variance and predictability of precipitation at seasonal-to-interannual timescales. J. Hydrometeor., 1, 26-46.

_ , P. A. Dirmeyer, A. N. Hahmann, R. Ipelaar, L. Tyahla, P. Cox, and M. J. Suarez, 2002: Comparing the degree of landatmosphere interaction in four atmospheric general circulation models. J. Hydrometeor., 3, 363-375.

- , and Coauthors, 2004: Regions of coupling between soil moisture and precipitation. Science, 305, 1138-1140.

— , and Coauthors, 2006: GLACE: The Global Land-Atmosphere Coupling Experiment. Part I: Overview. J. Hydrometeor., 7, 590-610.

Lewis, J. M., 2005: Roots of ensemble forecasting. Mon. Wea. Rev., 133, 1865-1885.

Lorenz, E. N., 1963: Deterministic nonperiodic flow. J. Atmos. Sci., 20, 130-141.

Miyakoda, K., J. Sirutis, and J. Ploshay, 1986: One-month forecast experiments-Without anomaly boundary forcings. Mon. Wea. Rev., 114, 2363-2401.

Murphy, J. M., 1988: The impact of ensemble forecasts of predictability. Quart. J. Roy. Meteor. Soc., 114, 463-493.

Numaguti, A., M. Takahashi, T. Nakajima, and A. Sumi, 1997: Description of CCSR/NIES atmospheric general circulation model. CGER's Supercomputer Monogr. Rep., No. 3, NIES, $1-48$.

Phelps, M. W., A. Kumar, and J. J. O'Brien, 2004: Potential predictability in the NCEP CPC dynamical seasonal forecast system. J. Climate, 17, 3775-3785.

Rowell, D. P., 1998: Assessing potential seasonal predictability with an ensemble of multidecadal GCM simulations. J. Climate, 11, 109-120.

— C. K. Folland, K. Maskell, and M. N. Ward, 1995: Variability of summer rainfall over tropical North Africa (1906-92): Observations and modeling. Quart. J. Roy. Meteor. Soc., 121, 699-704.

Scheffe, H., 1959: The Analysis of Variance. John Wiley and Sons, $477 \mathrm{pp}$.

Shukla, J., and Coauthors, 2000: Dynamical seasonal prediction. Bull. Amer. Meteor. Soc., 81, 2593-2606.

Stern, W., and K. Miyakoda, 1995: Feasibility of seasonal forecasts inferred from multiple GCM simulations. J. Climate, 8, 10711085.

Sugi, M., R. Kawamura, and N. Sato, 1997: A study of SST-forced variability and potential predictability of seasonal mean fields using the JMA global model. J. Meteor. Soc. Japan, 75, 717-736.

Taylor, G. I., 1920: Diffusion by continuous movements. Proc. London Math. Soc., 20, 196-212.

_ 1938: The spectrum of turbulence. Proc. Roy. Soc. London, A164, 476-490.

Thompson, P., 1957: Uncertainty of initial state as a factor in predictability of large-scale atmospheric flow patterns. Tellus, 9, 275-295.

Wiener, N., 1930: Generalized harmonic analysis. Acta Math., 55, $117-258$. 